\title{
Neue und alte Methoden der Behandlung der chirurgischen Tuberkulosen.
}

Von Dr. Wederhake, !andsturmpflichtiger Arzt, Facharzt für Chirurgie.

Die Behandlung der Tuberkulose der Weichteile, Knochen und Gelenke gehört zu den schwierigsten Aufgaben, die ein erfahrener Chirurg oft nur unter Aufopferung ganzer Glieder lösen kann. Hat die operative Chirurgie auf diesem Gebiete auch manchen Erfolg, so hat sie doch auch viele Nachteile, abgesehen davon, $\mathrm{da} ß$ gewisse Erkrankungen, z. B. die Tubcrkulose des Beckens und der Wirbelsäule, nicht radikal angegriffen werden können.

Tuberkulöse Lymphdrüsen z. B. am Halse zu exstirpieren ist undankbar. Hat die Röntgenbehandlung bei allen Drüsentuberkulosen doch bessere und schönere Resultate, als sie der sorg. fältigste und geschickt ausgeführte operative Eingriff haben kann.

Es ist nicht meine Absicht, weiter auf die Operation und konservative Behandlung näher einzugehen und das Für und Wider zu erörtern. Jeder erfahrene Chirurg hat seine Methoden, die ihn die praktische Erfahrung und die Kenntnis der Literatur gelehrt haben. Ob er mit ihnen zufrieden ist, muß er wissen.

Ich will nur einige Methoden beschreiben, die sich bei der Behandlung sehr zahlreicher chirurgischer Tuberkulosen bewährt haben. In der Zivilbevölkerung Russisch-Polens herrscht diese Erkrankung in erschreckendem Maße. Von russisch-polnischen Ärzten konnte ich keine Hilfe erwarten, da sie auf chirurgischem Gebiete nur wenig vorgebildet sind. Die Chirurgen waren von den Russen auf ihrem Rückzuge mitgenommen worden oder waren freiwillig nach Rußland gegangen. Mehr deutsche Ärzte zur Verfügung zu stellen, war nicht möglich. Mein Behandlungsgebiet umfaßte die Kinder und Erwachsenen in einem Industriegebiet Russisch-Polens, in welchem die Bevölkerung recht arm war und die hygienischen Verhältnisse erst durch die deutsche Verwaltung nach Kräften gebessert wurden, so wcit es in kurzer Zeit möglich 
war. Was in dicser knappen Zcit von $\mathrm{I}^{3} / 4$ Jahren auf hygienischem Gebiet für die russisch-polnische Bevölkerung geschehen ist, ist allerdings erstaunlich und gibt den deutschen Verwaltungsbehörden ein glänzendes Zcugnis. Die Verhältnisse eingchender zu schildern, liegt nicht in meiner $\Lambda$ bsicht. Ich möchte nur der veroreiteten Ansicht entgegentreten, als ob die jüdische Bevölkerung von der Tuberkulose der Knochen, Gelenke und Weichteile we.iger betroffen sei als die rein polnische Bevölkerung. Die Krankheit grassierte bei beiden Völkern in dem Bcobachtungsgebiet in gleicher Weise.

Wie bekannt, ist die Erkrankung der Weichteile, Knochen and Gelenke als eine S ch m u z k ra n kh e it aufzufasscn. Kleine Wunden an Füßen und Händen waren vielfach die Eingangs. Jforten für den Tuberkelbazillus, wie man an vielen Beispiclen besbachten konnte. Nicht der Blutweg führte meistens zur Ansteckung, sondern der Lymphweg. Von einer kleinen vernachässigten Wunde an einer Zehe zog sich der tuberkulös erkrankie Lymphstrang bis zu den Drüsen der Leiste und selbst bis in den Bauch hinein, oder die tuberkulöse Entzündung machte in einem ler Gelenke oder einem Knochen halt, lokalisierte sich hier oder ;chritt auch von hier wieder weiter. Uimgekehrt sprang die Tuberzulose nicht selten von dem Hüftgelenk zurück auf das Kniczelenk; oder die Wunden waren an den unteren Gliedteilen geveilt und die Lokalisation geschah erst in der Achsel. In anderen Fällen bestand eine Spina ventosa, eine zweite Lokalisation fand im Ellbogengelenk statt, die meistens von den Drüsen in der Nähe lieses Gelenks ihren Anfang genommen hatte. In anderen Fällen sestand die erste Etappe an der Wange, der Stirn, den Lippen usw. and die zweitc Etappe im Nacken, in der Halswirbelsäule usw.

$\mathrm{DaB}$ der Blutkreislauf nicht ausgeschaltet war bei der Versreitung der Tuberkulose, war oft zu sehen, so daß dic Tuberkulose fft alle Wcichteile der oberen und unteren Gliedmaßen ergriffen ratte.

In einer großen Zahl der Fälle suchte ich der weiteren Aussreitung der Tuberkulose durch Einspritzung von He tol Herr $: u$ werden. Ich hatte in einer Anzahl der Fälle sehr guten Erfolg sei den chirurgischen Tuberkulosen insofern, als die Tuberkulose 
durch die Hetoleinspritzungen günstig beeinflußt wurde. Örtlich reagierte das Gewebe mit guten frischen Granulationen und das tuberkulöse Gewebe schien zu heilen; aber nach einigen Einspritzungen hörte die Wirkung auf und wurde auch durch gesteigerte Dosen nicht wieder in Gang gebracht. Es wurden Dosen von $0,000 \mathrm{I}-0,05 \mathrm{Hetol}$ intravenös gebraucht. Nach meinen Beobachtungen liegt diese mangelhafte Wirkung des Hetols daran, daß es keinen Einfluß auf die Sekundärinfektion hat. Die Sekundärinfektion aber ist es, die der Verbreitung des Tuberkelbazillus immer wieder die Wege ebnet. Alle unsere Wunden, Fisteln usw. waren entsetzlich verschmutzt und eiterten stark, wenn sie in unsere Behandlung kamen. Diese Mischinfektion $z$ u bekämpfen, mußte unsere erste Aufgabe se in, wollten wir bei unseren Kranken zum Ziele kommen. Die ïblichen antiseptischen Maßnahmen versagten aber bei diesen Sekundärinfektionen vollständig. Wir mußten daher zu anderen Mitteln greifen und kamen damit zu dem von uns gesteckten Ziele.

Wenn wir uns vergegenwärtigen, wie schwer es ist, eine glatte Wunde von den Eitererregern zu befreien, so ist es erklärlich, warum diese Aufgabe bei einer buchtigen, durch Fisteln mit der Oberfläche kommunizierenden, viel verzweigten tuberkulösen Höhle nicht zu lösen ist. Kein Antiseptikum ist imstande, eine solche Höhle zu desinfizieren, wenn es nicht besondere Eigenschaften hat. Hier versagte auch das Jodoform fast vollständig, da seine Einwirkung auf die Eitererreger nur sehr gering ist. So vor$z$ üglich das Jodoform auf das tuberkulöse Gewebe und a f die Tuberkelbazillen wirkt, sowenig vermag es a uf die Sekundärinfektion $z u$ wirken. Das konnte man gut an den geschlossenen Weichteilabszessen, wie sie sich besonders als Senkungsabszesse zeigen, beobachten. Punktiert man einen solchen Abszeb und injiziert man Jodoform, z. B. Io Proz. Jodoformglyzerin, so heilen eine Anzaht đieser $\mathrm{Ab}$ szesse in einigen Wochen; viele tuberkulöse Abszesse aber ver halten sich refraktär. Es kommt bei ihnen doch zum Durchbruch; es entsteht eine Fistel und der schon bestehenden Sekundär. infektion ist ein neues Tor geöffnet. Die ubiquitären Eitererreger bemächtigen sich der sezernierenden Fistel sofort und in kurzer Zeit ist die Sekundärinfektion gesteigert, ohne daß das in dem 
Abszeß enthaltene Jodoform die Eitererreger am Eindringen und Wachsen und Vermehren hindert.

Das war der Angelpunkt, wo wir eingriffen. Es mußten drei Dinge geschehen, um den genannten Übelständen entgegenzutreten:

1. Es war notwendig, die in dem geschlossenen tuberkulösem Abszesse 'lebenden Tuberkelbazillen zu töten und so die Grundursache zu beseitigen.

2. Die im geschlossenen Abszesse lebenden Eitererreger muß. ten abgetötet werden.

3. Der Verbreitung der Eitererreger durch Lymph- und Blutbahn mußte entgegengetreten werden, damit sie sich nicht an einer anderen Stelle festsetzen konnten und einen neuen locus minoris resistentiae für die Ansiedlung der sich im Körper aufhaltenden Tuberkelbazillen, die durch das Tuberkelbazillen tötende Mittel nicht erreicht wurden, schaffen konnten.

Diese drei Aufgaben haben wir durch unsere Behandlung gelöst, wie unsere zahlreichen guten Erfolge bewiesen. Diese Erwägungen sind nicht aus der Theorie hervorgegangen, sondern wurden uns durch die klinische Beobachtung aufgedrängt. Die Wege, die wir einschlugen, waren folgende:

I. Um dic Tuberkelbazillen in der Abszeßhöhle abzutöten, wurde diese punkticrt, ein Teil des mehr oder weniger flüssigen Eiters abgelassen und die Höhle reichlich mit ıo proz. Jodoformglyzerin gefüllt. Dieses blieb drei Tage in der Höhle.

2. Nach Ablauf dieser drei Tage wurde von ncucm punktiert, wieder ein Teil des Eiters, der jetzt etwas dünnflüssiger war, entleert, wiederum mit Jodoformglyzerin eingcfüllt, jedoch nur so weit, daß die Wand nicht unter Druck stand und noch so viel Raum vorhanden war, daß je nach der Größe der Höhle noch $1--5 \mathrm{ccm}$ einer wässerigen 5 proz. Lösung von Acid. tannicum cingefüllt werden konntc, ohne daß auch jetzt die Wand unter Druck stand. Sowie dic Tanninlösung eingespritzt ist, erstarrt der größte 'Teil des Eiters zu einer steifen Masse, die aus zusammengeballtcn Eiterkörperchen, Fibrin, Bakterien und Jodoform besteht.

Zwei Stunden nach der Tannineinspritzung wird der AbszeB durch eine kleine Stichincision eröffnct. Es entleert sich hierbei 
fast nur einc seröse, etwas braun gefärbte Flüssigkeit ohne Beimischung von größeren Mengen Eiter.

Dic Wände des Abszesses werden leicht angedrückt, aber der Abszeß nicht ,ausgedrückt“. Die kleine Stichwunde wird „,dick“" mit Jodoformpulver bedeckt, so daß keine Lücke von Jodoform frei ist. Das Jodoform bedeckt jedoch nur die Wunde selbst und nur etwa $0,3 \mathrm{~cm}$ des Wundrandes, nicht die ganze Abszeßwand. Auf das Jodoform wird ein kleiner Tupfer aus Mull gelegt, welcher in eine 5 proz. wässerige Tanninlösung getaucht wird und tricfend naß, also ohne Ausdrücken, mit einer Fasse (anatomischen Pinzette) aufgelegt wird. Darüber wird ctwas Mull gelegt und das ganze durch einen Mullschleier mit Mastix rings festgeklebt. Dieses letztere muß besonders sorgfältig und exakt geschehen, damit eine Verschiebung des Tannintupfers von der Wunde unter keinen Umständen geschchen kann. Die Garantic für das exakte Festhalten des Tupfers bedingt die Garantie für die Heilung.

Nächster Verbandwechsel nach drei Tagen. Wiederbedecken der Wunde dick mit Jodoform, darüber Tannintupfer, Fixieren mittels Mullmastixschleiers. Nächster Verbandwechsel wieder nach drei Tagen wie vorhin und so fortfahrend bis zur Heilung.

$\mathrm{Nicmals}$ wird ein tuberkulöser AbszcB drai. niert, niemals tamponiert, niemals ein Jodoformstrcifen eingelegt. So wurden alle geschlossenen tuberkulösen Abszesse behandelt, einerlei, ob sie ihren Ursprung im Knochen, in den Drüsen oder in den Weichteilen hatten.

Wie wirkt nun die Behandlung? Die fast spezifische Wirkung des Jodoforms auf die chirurgischen Tuberkulosen ist bekannt und bedarf keiner weiteren Erklärung. Das Jodoform wirkt aber nur in geschlossenen Höhlen. Es ist in seiner spezifischen Wirkung durch kein anderes Mittel, weder Airol, Xeroform, Dermatol usw., noch durch Lugolsche Lösung, noch durch verdünnte Jodtinktur usw. zu crsetzen.

Sobald zu dem Abszeßeiter Tannin gefügt wird, gerinnt, wie erwähnt, der Eiter und ballt sich zu einer festen Massc, aus Eitcrkörperchen, Fibrin, Bakterien und Jodoform bestehend, zusammen, wobei die im Eiter vorhandene Flüssigkeit ausgepreßt wird. Das Tannin tötet sämtliche Eitererreger ab: Es findet also eine vollständige Sterilisation der Eiterhöhle und ihrer Gänge statt. 
Gleichzeitig werden die Granulationen, die bei der Tuberkulose fast immer sehr schlaff sind, durch die adstringierende Wirkung zusammengezogen und ihrer Flüssigkeit beraubt. Sie werden fest und widerstandsfähig. Die Tannineinspritzung ist nur wenig schmerzhaft. Fast unmittclbar nach der Injektion tritt eine sehr heftige Hyperämie ein, die sich bis ticf in die Umgebung des Abszesses erstreckt. Würde man die Tanninlösung mehrere Tage in der Abszeßhöhle lassen, so würde diesc sich prall mit Flüssigkeit füllen, so daß die darüber liegende Haut gespannt wäre und die Flüssigkeit sich cinen Weg durch die Haut bahnen würde. Das soll vermieden werden. Daher wird der wässerigen Flüssigkeit bereits nach zwei Stunden durch Stichöffnung ein Abfluß gewährt. Der Schnitt soll aber nicht größcr sein, als zur Entleerung erforderlich ist, damit Narben möglichst vermieden werden und die Höhle sicher durch Jodoformpulver geschlossen gehalten werden kann, ohne den Sekretabfluß zu behindern.

Alle unsere Maßnahmen geschehen selbstverständlich nach strengen Regeln der Ascpsis, denn eine Neuinfektion der Höhle mit Eitererregern muß unter allen Umständen vermicden werden. Die Eröffnung des tanninisierten Abszesses soll durch Stich mit dem Messer und nicht durch Punktion geschehen, um ein Wiederansammeln unter allen Umständen zu vermeiden und den weiteren Abfluß des Sekretes sicher zu stellen.

Würde die Entleerung des tanninisierten Abszesses nur durch Punktion geschehcn, so würde sich Sekret wieder ansammeln und einen Druck auf dic Abszeßmembran und ihre Umgebung ausüben und diese Gewebe in ihren Heilungsbestrebungen stören, was wir durchaus vermciden wollen. Im Gegenteil müssen wir diese Heilungsbestrebungen mit allen Mitteln unterstützen. Darin hilft uns auch der in der Abszeßhöhle zurückgebliebene Jodoformfibrinblock, welcher einerseits von den umliegenden Geweben aufgezehrt und anderseits zum Aufbau und zur Ausfüllung der Höhle dient, indem er von den umliegenden Granulationen durchwuchert wird. Er kann aber von den etwa noch vorhandenen Tubcrkelbazillen wegen des darin enthaltenen Jodoforms nicht zur Ansiedlung benutzt werden; er wirkt auf die Tuberkelbazillen tötend und hemmt die in ihrer Lebensfähigkeit geschwächten Tuberkelbazillen weiter in ihrer Entwicklung. Es wäre des. 
wegen ein Fehler, wenn wir diesen Jodoformfibrinblock etwa mit dem scharfen Löffelentfernen wollten. Ein Auskratzen oder Ausätzen, eine An. wendung des Höllensteinstiftes usw. muB unter allen Umständen vermieden werden und würde unsere Methode nur in ihrer Wirksamkcit schädigen und stören. Ein jeder tuberkulöser Abszeß is t alsoindicser Hinsichtein nolime tangere.

Überlassen wir die weitere Heilung der Natur und geben wir der geöffneten Höhlc nur einen sicheren Verschluß durch Jodoformpulver, halten wir die Bakterien in der dic Öffnung umgebenden Haut durch einen Tannintupfer nieder, so heilt der AbszeBsicherohnc weitere Eingriffe.

Nicmals werden auch bei tuberkulösen Ab. szessen der Knochen Sequester entfernt, weder durch denscharfen Löffel, nochdurchden Meißel. Die tuberkulösen Abszesse der Knochen heilen unter der geschil. derten Behandlung genau so gut wic die Abszesse der Weichteile. Kleinere Sequester stoßen sich gelegentlich mit aus. Größere Sequester heilen fast immer reaktionslos ein, wenn nur die große Sterilisation des $\Lambda$ bszesses gelungen ist, und sic gelingt stets, wenn sie nach den gegcbenen Regeln geschieht: Ich kenne bei vielen hundert Knochentuberkulosen, die ich behandelt habe, keinen Fall, bei welchem ich gezwungen gewesen wäre, zum scharfen Löffel oder zum Meißel zu grcifen. Der Meißel wie der scharfe Löffel muB aus unscrem Rüstzeug zur Bekämpfung der Knochentuberkulose verbannt werden.

$\mathrm{Zu}$ bemerken ist noch, daß über den nassen Tannintupfer niemals undurchlässiger Stoff gelegt werden darf.

Daß das Tannin ein hervorragendes Mittel zur Abtötung der Eitererreger, besonders der Streptokokken ist, habe ich bereits in frütheren Arbeiten ausgeführt (W e d e r h a k c, Ein Ersatz der Jodtinktur. Münchn. med. Wochenschr. 1917, Nr. 24, Sch merz I916, ebenda). Das Tannin selbst heilt keine chirurgische Tuberkulose, wie ich das bereits früher ausgeführt habe. Aber es unterstützt unser therapeutisches Handeln durch Sterilisation der Abszeßhöhle, durch Schaffung des Jodoformfibrinblocks, durch 
Schrumpfung der schlaffen tuberkulösen Granulationen und Fernhaltung der Sekundärinfektion, die der geöffneten sterilisierten Höhle droht.

3. Aber wir müssen noch einen Schritt weiter gehen. Ist eine Sekundärinfektion eines tuberkulösen geschlossenen Herdes geschehen, so enthalten die umgebenden Gewebe mehr oder weniger Eitererreger, die nur mobilisiert zu werden brauchen, um wieder neue Abszesse zu schaffen und den Tuberkelbazillen, die in der Nähe leben, einen Ort zur gedeihlichen Wuchcrung zu schaffen. Diese Eitererreger und Tuberkelbazillen sind durch die Sterilisation der Abszeßhöhle mit Jodoform und Tannin nicht zu erreichen. $\mathrm{Da}$ muß anders angegriffen werden. Mit den Tuberkelbazillen an sich würde der Organisums wohl meistens fertig werden; aber gegen die Eitererreger fehlen ihm die nö:igen Hilfsmittel. Wir müssen ihm diese zuführen und haben ein ausgezeichnetes Mittel sowohl gegen Staphylo- als Streptokokken in den Terpenen des Terpentinöls und des Kampfers. Das Terpentinöl kann fast als diagnostisches Mittel verwendet werden, um festzustellen, ob einc Mischinfektion vorliegt oder nicht. Injizieren wir einem Kranken mit Mischinfektion $0,3 \mathrm{Ol}$. terebinthinac in die Gesäßmus. kulatur, so steigert sich in den nächsten 4-5 Tagen die Abendtemperatur um $0,6-1,6 \mathrm{Grad}$, um nach dieser Zeit wieder auf die frühere Höhe zu fallen. Wir gebrauchen zur Injektion eine zo proz. Lösung von Ol. tercbinthinac in Ol. olivarum oder Ol. arachidis oder Ol. amygdal., selten in Ol. rapae, da letzteres (il etwas reizt. Nach der Injektion bleiben die Kranken 5 Tage zu Bett liegen. Bei denjenigen Kranken, die an Mischinfektion leiden, tritt häufig an der Injektionsstelle (Gesäßmuskulatur) einc lokale Reizung auf, die je nach der Schwere der Mischinfektion einen höheren oder geringeren Grad crreicht, immer aber in einigen Tagen abklingt (örtliche Reaktion auf Mischinfektion). Sollte die lokale Reizung zu einer starken Schwellung der eingespritzten Stelle führen, was selten ist, so sind Umschläge mit verdünnter essigsaurer Tonerde zweckmäßig und lindern das etwa auftretende Spannungsgefühl. Tritt garkeine Reizerschein ung a uf (weder Fieber noch örtliche Reaktion), so liegt nach unseren Erfahrungen keine Mischinfektion vor. 
Wir spritzen meistens $0, \mathrm{I}-0,3 \mathrm{~g}$ Ol. terebinthinae intrag l u t ä a l ein, ohne je Reizung der Nieren zu sehen. In den meisten Fällen wurde der Urin vor und nach der Einspritzung auf Eiweiß untersucht. Es ist ganz erstaunlich zu beobachten, wie die Mischinfektion schwindet und keine neucn Herde wieder auftreten.

Das Bestreuen der Stichöffnungen mit dickem Jodoformwall muß bisweilen cinige Tage ausgesetzt werden, da das Jodoform die Umgebung der Stichöffnung reizt; einfacher Verband mit 5 proz. wässeriger Tanninlösung ohne Jodoform beseitigt diesen Übelstand in einigen Tagen.

Die Zahl der gegebenen Terpentineinspritzungen schwankt zwischen 5 und 70 .

Ganz ähnlich behandelten wir die schon mit Fisteln einhergehenden chirurgischen Tuberkulosen: Auch hier kam es uns in erster Linie darauf an, die Mischinfektion zu bekämpfen und gleichzeitig den tuberkulösen.Herd anzugreifen. Zu diesem Zwecke spritzten wir mit einer Io $\mathrm{ccm}$ fassenden Rekordspritze langsam und ohnc Druck 5 proz. wässerige Tanninlösung in die Fisteln; dann plombierten wir dic Fistelöffnung mit Jodoformpulver und legten darüber einen Tupfer mit wässeriger Tanninlösung, wodurch die auf der Haut der Umgebung auftretenden, durch das Jodoformpulver und den Fisteleiter bedingten Reizerscheinungen (Ekzeme) bekämpft wurden. Sind es doch gerade diese Reizerscheinungen der Haut, welche die Sekundärinfektion befördern und unterhalten. Die Erfolge waren gut bei solchen Fisteln, welche nicht von Opcrationen herrührten. Dicse letzteren waren besonders schwer zu bekämpfen, wie ja die Operation - namentlich wenn sie unvollkommen ist - die Heiiung nicht schnellererzielen kann als unser kon. servatives Verfahren.

Injizicrt man in eine tuberkulöse Fistel Jodoformglyzerin, so nimmt die Sekretion zu. Dieses ist nicht erwünscht, weil durch sie die Gefahr der Mischinfektion gestcigert wird. Wir bekämpfen diese vermehrte Sekretion der Fisteln durch das Durchspülen mit wässeriger 5 proz. Tanninlösung und durch intramuskuläre Injektion von Terpentinöl :und schränken dadurch die Sekundärinfektion ein.

Nur bei ganz offen liegenden tuberkulösen Herden, welche 
keine Fisteln haben, die in die Tiefe führen, wie Lupus, Tuberkulose des Stumpfes nach Amputationen usw., wenden wir ein anderes Verfahren an, um schneller zum Zie!e zu kommen.

Bepinseln wir eine solche Stelle mit 5 proz. wässeriger 'Tanninlösung ziemlich reichlich und betupfen sie darauf mit konzentrierter wässeriger Kalium hypermanganicum-Lösung, so erzeugen wir auf der Wunde Py rogallol, welches bekanntlich eine elek. tive Wirkung auf das tuberkulöse Gewebe ausübt. Diese Wirkung wissen wir seit langem bei der Behandlung des Lupus auszunutzen. Lassen wir einen Verband auf einer tuberkulösen Wunde, welche in dieser Weise behandelt wurde, einen Tag liegen, so setzt eine mächtige Eitersckretion cin; in den Granulationen sind die tuberkulösen Knötchen als solche zu erkennen; bringen wir das Verfahren zum zweiten Male zur Anwendung, so werden diese tubcrkulösen Gewebe zerstört, wobei das in statu nascendi befindliche Pyrogallol seine große elektive Wirkung besonders entfalten kann.

Die chemische Wirkung können wir uns folgendermaßen vorstellen: Tannin ist polymer, aber nicht identisch mit Digallussäure, welche die Formel $\mathrm{C}_{6} \mathrm{H}_{2}(\mathrm{OH})_{3} . \mathrm{CO} . \mathrm{OC}_{6} \mathrm{H}_{2}(\mathrm{OH})_{2} \mathrm{COOH}$ hat. Spalten wir von der Digallussäure Pyrogallol $a b$, so erhalten wir folgende Reaktion:

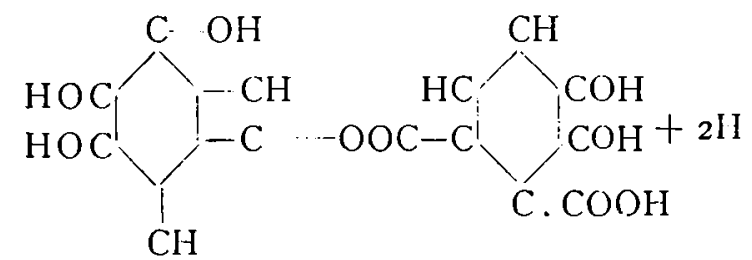

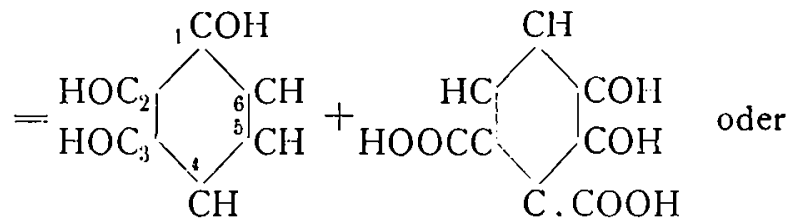

${ }_{2}(\mathrm{OH})_{3} \cdot \mathrm{CO} \cdot \mathrm{OC}_{6} \mathrm{H}_{2}(\mathrm{OH})_{2} \cdot+2 \mathrm{H}=\mathrm{C}_{6} \mathrm{H}_{3}(\mathrm{OH})_{3}+\mathrm{C}_{6} \mathrm{HO}_{2}(\mathrm{OH})_{2}<\mathrm{COOH}$

Digallussäure =: Pyrogallol - Dioxybenzocdikarbonsäure.

Pyrogallol= Pyrogallussäure $=$ Trioxybenzol ist cin clreiwertiger $\Lambda$ kohol, weiße, in Wasser leicht lösliche Blättchen, dic kräftig reduz i e re nd wirken und in alkalischer Lösung begierig Sauerstoff anziehen (absorbieren). 
Auf diesen Eigenschaften beruht dic elektive Zerstörung der Tuberkelknötchen, die wir uns bei unserer Behandlung der offenen Tuberkulose zunutze machen. Um aber die stark eitererregende Wirkung des entstehenden Pyrogallols zu bekämpfen, tragen wir einen Überschul von wässeriger Tanninlösung auf. Die Behandlung ist weniger schmerzhaft, als wenn wir zur Beschränkung der Sekretion Sublimatumschläge verordnen würden, wie sic in der Dermatologic üblich sind.

Die: Dermatologen benutzen das Pyrogallol in Io proz. Salbe vielfach zur Zerstörung von Lupusknötchen. Die nach dieser Salbe einsetzende starkc Sekretion bckämpft man mit Umschlägen von vcrdünntem Sublimat. Hebt man einen solchen Umschlag an einer Stelle etwas auf, so daß die Luft hinzutreten kann, so ist diese Stelle sehr schmerzhaft. Das vermeiden wir, wenn wir von vornherein einen feuchten, mit wässcriger Tanninlösung gctränkten Tupfer auflegen oder Tanninpulver aufstreuen.

Bei der Abspaltung des Pyrogallols aus dem Tannin entsteht die Dioxybenzoedikarbonsäure; aus dieser gehen unter Abspaltung von Kohlensäure dic Dioxybenzole: Brenzcatechin, Resorcin, Hydrochinon hervor:

$$
\mathrm{C}_{6} \mathrm{H}_{2}(\mathrm{OH})_{2}<\mathrm{COOH} \longrightarrow \mathrm{C}_{6} \mathrm{H}_{4}(\mathrm{OH})_{2}+._{2}\left(\mathrm{COO}_{2} .\right.
$$

Daß Resorcin bei örtlicher Anwendung günstig auf die Gewebstuberkulose wirkt, wissen wir aus der Behandlung des I.upus mit Resorcinsalben.

Durch Reduktion entsteht aus der Dioxybenzoedikarbonsäure die Monooxybenzoekarbonsäure

$$
\mathrm{C}_{6} \mathrm{H}_{2}(\mathrm{OH})_{2}<\mathrm{COOH}+-2 \mathrm{H} \longrightarrow \mathrm{C}_{6} \mathrm{H}_{4} . \mathrm{OH} . \mathrm{COOH} ; \mathrm{H}_{2} \mathrm{O}_{-i} \mathrm{COO}_{2} \text {. }
$$

Dic: Orthomonoxybenzockarbonsäure $\left(\mathrm{C}_{6} \mathrm{H}_{4} \mathrm{OH} . \mathrm{COOH}\right)$ ist die Salizylsäure, ein Antiseptikum, welches in statu nascendi besonders wirksam ist und dic Mischinfektion gut bekämpft.

Aus der Dioxybenzoedikarbonsäure kann aber auch durch Reduktion dic Orthobenzocdikarbonsäure entstehen:

$$
\mathrm{C}_{6} \mathrm{H}_{2}(\mathrm{OH})_{22}<\mathrm{COOH}^{\mathrm{COOH}}{ }_{4} \mathrm{H} \longrightarrow \mathrm{C}_{6} \mathrm{H}_{4}<\mathrm{COOH}^{\mathrm{COOH}}{ }^{-}{ }_{2} \mathrm{H}_{2} \mathrm{O} \text {. }
$$

Die Orthobenzocdikarbonsäure heilit auch Phthalsäure, 
die bekanntlich stark blutstillende Eigenschaften hat. Sic läßt sich auch durch Oxydation mit Naphthalin darstellen. Aus der Phthalsäure läßt sich durch Abspalten von Kohlensäure dic Benzoesäure $\mathrm{C}_{6} \mathrm{H}_{5} \mathrm{COOH}$ herstellen.

$$
\mathrm{C}_{6} \mathrm{H}_{4}<\underset{\mathrm{COOH}}{\mathrm{COOH}} \longrightarrow \mathrm{C}_{6} \mathrm{H}_{i,} \cdot \mathrm{COOHI}+\mathrm{CO}_{2} \text {. }
$$

Dic Benzoesäure ist wiederum ein $\mathrm{g} u$ t cs Antiscptikum. Das Tannin geht durch Hydrolyse in Gallussäure, welche Trioxybenzockarbonsäure $\mathrm{C}_{6} \mathrm{H}_{2}<\mathrm{COOH}$ (OH $)_{; j}$ ist, über. Diese gibt unter Abspaltung von Kohlensäure cbenfalls Pyrogallol:

$$
\mathrm{C}_{6} \mathrm{H}_{2}<\underset{\mathrm{COOH}}{(\mathrm{OH})_{3}} \rightarrow \mathrm{C}_{6} \mathrm{H}_{3}(\mathrm{OH})_{3}--\mathrm{CO}_{2} .
$$

Die Dioxybenzoekarbonsäurc, das eben erwähnte Spaltungsproduki des Tannins, ergibt beim Abspalten von 2 Molckülen Kohlensäure das Dioxybenzol (Brenzcatechin, Rcsorcin, $\mathrm{Hy}$ drochinon)

$$
\mathrm{C}_{6} \mathrm{H}_{2}(\mathrm{OH})_{2}<\mathrm{COOH} \longrightarrow \mathrm{C}_{6} \mathrm{H}_{4}(\mathrm{OH})_{2}+2 \mathrm{COOH}_{2} \text {. }
$$

Uxydieren wir dic Ijoxybenzoedikarbonsäure, so erhalten wir wieder Pyrogallol

$$
\mathrm{C}_{6} \mathrm{H}_{2}(\mathrm{OII})_{2}<\underset{\mathrm{COOH}}{\mathrm{COOH}} \longrightarrow \mathrm{C}_{6} \mathrm{H}_{3}(\mathrm{OH})_{3}+\mathrm{O}_{2} \mathrm{CO}_{2} \text {. }
$$

Aber wir können aus Tanmin selbst durch Hydrolyse Py rogallol erhalten:

$$
\begin{aligned}
& \mathrm{C}_{6} \mathrm{H}_{2}(\mathrm{OH})_{3} \cdot \mathrm{CO} . \mathrm{OC}_{6} \mathrm{H}_{2}(\mathrm{OHI})_{2} \cdot \mathrm{COOH}+\mathrm{H}_{2} \mathrm{O} \longrightarrow 2 \mathrm{C}_{6} \mathrm{H}_{3}(\mathrm{OH})_{3}+ \\
& { }_{2} \mathrm{CO}_{2} \text {. }
\end{aligned}
$$

Wir sehen also, daß wir es bei den Spaltprodukten des Tannins mit sehr reaktionsfähigen Körpern zu tun haben, die als ausgezeichnete Arzneimittcl bekannt sind.

Wir begegnen dem Pyrogallol, welches sich auf verschicdenen Wegen aus dem Tannin abspaltet; dieses Heilmittel wirkt als Spezifikum bei der örtlichen Anwendung auf $t u$. berkulösc Gewebe; nach Ansicht vieler erfahrener Hautärzte ist das Pyrogallol fast das cinzige Arzneimittel, mit welchem man gute Dauercrfolge bei Lupus eraiclen kann. Wir begegnen weiter dem Rcsorcin, einem vorzüglichen Ätzmittel, Adstringens und Desinfizicns, das ebenfalls vielfach in der Dermatologic Verwendung 
finder. Wir sehen die Salizylsäure und die Benzoesäure entstehen, die wiederum wertvolle lokal wirkende Eigenschaften als Antiseptica haben.

Die Gallussäurce und I'hthalsäure haben cbenfalls Eigenschaften, die wir zu schätzen wissen, da sie verhindern, daß die tuberkulösen Granulationen schlaff sind und leicht bluten; die kleinen $13 \mathrm{l}$ ut ungen der Granulationen sind aber häufig eine Ursache neuer Infektion, sowohl bei der Verbreitung der primären tuberkulösen Erkrankung, wie bei der Mischinfektion.

Nicht zu unterschätzen ist auch die frci werdende Kohlensä ure, welche \%war nur wenig antiseptisch wirkt, aber den Tu. berkelbazillen doch das Leben sauer macht, so daß sie schlechter gedeihen.

Wir haben es aber auch in der Hand, dasjenige Mittel aus dcm 'Tannin auf der tubcrkulösen Wunde selbst entstehen zu lassen, welches für den gegebenen Fall besonders gceignct ist. Pyrogallol und Gallussäure erhalten wir durch Iydrolyse oder durch Oxydation (Kal. hypermanganicum, chlorsaures Kalium usw.), Phthalsäure, Dioxybenzoedikarbonsäure, Resorcin, Salizylsäure und Benzoesäure erzielen wir durch die Reduktion (Wasserstoffsuperoxyd, Zucker, durch das in den lebenden (jeweben und Säften vorhandene Ferment, die Katalase). Daß diese Vorgänge im lebenden Eiter und Gewebe sich gleichzeitig abspielen, begünstigt unsere Absichten. WirbrauchendicReduktionnichtkünstlich hervorzurufen, da in den lebenden lieweben dicser Vorgang durch die Katalase zustande kommt. Die Oxydation haben wir unwillkürlich in unserer Hand und wenden sie ganz nach Be. darfan.

Der Hauptbestandteil des Terpentinöls ist das $P$ in e $n$, wel ches ein Ierpen $\mathrm{C}_{10} \mathrm{H}_{16}$ ist, ein ungesättigter Kohlenwasserstoff mit cinem sechsgliedrigen geschlossenen Ring, leicht oxydierbar. Der wichtigste Angehörige der Terpengruppe ist der $\mathrm{Kam}$ p f e r C $\mathrm{C}_{10} \mathrm{H}_{16}(\mathrm{O}$. Sowohl das 'Terpentinöl wie der Kampfer unterstützen die Heilungsbestrebungen bei den chirurgischen $\mathrm{Tu}$ berkulosen ganz wesentlich, da der Kampfer durch Oxydation in $\mathrm{Zimm}$ s ä ur e $\mathrm{C}_{10} \mathrm{H}_{16}$. CH: CH. COOH übergeht.

$$
\mathrm{C}_{10} \mathrm{H}_{16} \mathrm{O}+6 \mathrm{O} \longrightarrow \mathrm{C}_{6} \mathrm{H}_{5} . \mathrm{CH}: \mathrm{CH} .\left(\mathrm{COH}-3 \mathrm{H}_{2} \mathrm{O}+\mathrm{CO}_{2}\right. \text {. }
$$


Dic Zimmtsäure verwenden wir in Form des zimmtsauren Natrons(Hetollanderer)schonlange zur Behandlung der Lungentuberkulose, wie den Kampferselbst. Aber a uch das Pinen, der Haupt. bestandteil des Terpentinöls, geht durch Oxydation in Zimmtsäure über.

$\mathrm{C}_{10} \mathrm{H}_{16}+8 \mathrm{O} \longrightarrow \mathrm{C}_{6 i} \mathrm{H}_{5} . \mathrm{CH}: \mathrm{CH} . \mathrm{COOH}+4 \mathrm{H}_{2} \mathrm{O}-\mathrm{i}-\mathrm{CO}_{2}$.

Daß das zimmtsaure Natron einen spezifisch heilenden Ein fluß auf das tuberkulösc Gewebe ausübt, ist bei den offenen tuberkulösen Erkrankungen leicht zu beobachten. Nach intravenöser Einspritzung von Hetol erscheinen die (icwebc frischrot; gute rot: Granulationen liegen vor uns. T)a wir aber nicht längere Zeit Kampfer geben können, so können wir leicht mit Hetol- und Terpentinölinjektionen abwechseln. Vor den Kampfereinspritzun. gen hai dic Terpentinöleinspritzung den großen Vorteil, daß si: in $\mathrm{ramuskulär}$ gegeben werden kann, und dal3 die Jestandteile des Terpentinöls günstig auf die Mischinfektion, besonders dic Staphylokokken wirken.

Die Trias zimmtsaures Natron (Hetol), Terpentinöl und Kamp. fer darf aus unseren Mitteln zur Bekämpfung der chirurgischeri Tuberkulose nicht mehr verschwinden. Sie inüssen uns helfen, das tuberkulöse Gewebe in seincn Heilungsbestrebungen zu unterstützen und die Mischinfektion, die Hauptursache der Weiterverbreitung der Luberkulösen Prozesse im Körper, zu bekämpfen.

Die viel gelobte und vicl geschmähte. Hetolbehandlung nach Landerer hat zweifellos ihre Berechtigung. Sie ist aber nur selten alle in imstande, eine chirurgische Tuberkulose zur Heilung zu bringen, da diese außer der Allgemeinbehandlung der örtlichen Behandlung bedarf. Ohne örtliche Behandlung dauert die Iletolkur sehr lange und führt nur in einem Teil der f:älle zur Heilung.

Unsere Behandlungsmethode macht die sonst üblichen Heilarten, wic Höhenluft, Freiluft, Mastkur, Licgekur, Sonnenbehandlung. Lichtbehandlung, Solbäder, Röntgenbestrahlung usw. nicht überflüssig, sondern sie bedient sich ihrer mit großem Vorteil und zieht aus diesen Mitteln den Nutzen der allgemeinen Kräftigung des kranken Körpers. Wir haben unsere Behandlung bis zur Klärung der Sachlage ohne diese. Mittel durchgeführt; ich zögere 
aber nicht, sie anzuwenden, um noch schneller zum Zicle zu kommen und dic Ileilung zu sichern.

Die Ungunst der Vcrhältnisse zwang uns, auf diese Mittel vorläufig zu verzichten; auch in Zukunft müssen wir zum 'Teil ohne sic auskommen. Denn cinerseits gehörten unsere Kranken, wie crwähnt, meistens den ärneren Ständen an, andererscits stehen keine Mittel zur Verfügung, dic genannten Heilmethoden in gröBerem Vaßstabe zu gebrauchen. Dazu kommt, daß der russische Winter schr lang, daß dic Zahl der Sonnentage sehr gering ist und dal' ein großer Teil der Kranken ambulant behandelt werden muß. Rechnen wir dazu die Unvernunft und das geringe Verständnis der russisch-polnischen Bevölkerung für unsere Bestrebungen, so ist es erklärlich, daß die Schwierigkeit, die reborierende Behandlung durchzuführen, grol3 ist. Geschieht auch von der deutschen Verwaltung alles, was in Menschenkräften steht, so sind wir doch nicht imstande, viele Schädigungen abzuhalten, zumal die hygienischen Verhältnisse (Wohnung, I.cbensweise usw.) die denkbar schlechtesten sind und ihre Besserung erst seit kurzem aufgenommen ist. Wenn wir trot $z$ allem gute lerfolge hatten, so mub dic Nethode sehr leistungsfähig scin; aus diesem Grunde übergebe ich sie zur Nachprüfung der Offentlichkeit.

Ich sche davon ab, einzclne Krankengreschichten zu geben, da sic bei der Fülle des Materials nur ein unvollkommenes Bild geben können. Dic bchandelten Fälle sämtlich vorzuführen, ist bei der großen Zahl wegen Nangel an Raum und Arbeitskräften unmöglich.

Dic Literatur anzuführen, liegt nicht in meiner Absicht, sic: ist dem Bewanderten hinreichend bekannt und kann ihm nichts Neues bringen, den weniger Bewanderten würde sie nur verwirren. Dazu kommt, daß es mir nicht möglich ist, sie mir hier im Felde lückenlos zu beschaffen; lückenhafte Literaturangaben haben wenig Wert. Zur gecigneten Zeit hoffe ich, darauf cingehend zurück. kommen zu können.

Unsere konservative Behandlung ist nicht in allen Phasen neu; sie bcnutzt viclmehr dic vorhandenen Erfahrungen in geeigneter Weisc. Sie ist überall da anwendbar, wo der Körper noch eine Behandlung von einigen Wochen aushalten kann, ohne an seinem Leiden zugrunde zu gehen. Da es uns gelingt, bei reaktions - 
fähigem Körper die chirurgische Tuberkulose sicher zu heilen, so kann die rein operative Behandlung nur in Frage kommen, wenn es sich um eine Indicatio vital is handelt. Diese ist nur bei sehr dekrepiden Kranken gegeben, bei denen die radikale Wegnahme des gesamten tuberkulösen Herdes möglich und nötig ist. Es kann sich hierbei nur um Amputation und Resektion handeln.

Keine tuberkulöse Fistel, kein Knochenherd, keine Gelenkentzündung sollte operativ angegangen werden, wenn der Körper noch reaktionsfähig ist. Sie sind konservativ zu behandeln und kommen bei konsequentem Handeln sicher zur Heilung unter der genannten Voraussetzung.

Kontrakturen sollen durch vorsichtig dosierte Streck. verbände bescitigt werden; eine gewaltsame Streckung in Narkose ist stets von Übel. Besser ist es sogar, die Heilung in Kontraktur herbeizuführen, wenn dic Kontraktur nicht durch cinfache Streckverbände beseitigt oder verhütet werden kann, was bei sorgfältiger Behandlung selten ist. J)ie Korrektur der Kon. trakturist dann nachvollständiger Heilung durch passende operative MaBnahmen, nicht durch Re. dressement vorzunchmen. Sie soll nicht unmittelbar im AnschluB an die Heilung des tuberkulösen Prozesses gemacht werden, sondern erst nach Ablauf einer gewissen Frist, dic ein jeder erfahrene Chirurg bestimmen kann.

Ich stimme $\mathrm{W}$ il ms durchaus bei, wenn er und mit ihm viele andere raten, die tubcrkulösen Drüsenerkrankungen, besonders des Halses, durch Röntgenbestrahlung zu heilen. Exstirpieren wir sie, so haben wir als Folge nicht nur die häßliche Narbe, wir crleben auch bei radikalstem Vorgehen $\mathrm{Rezidive;} \mathrm{abgesehen}$ davon, daß der Eingriff bei schr großen Drüsenpaketen sehr eingreifend ist.

Stehen uns Röntgenstrahlen nicht zur Verfügung, so müssen wir auf diese idcalc Ieilungsmethode verzichten; ich rate aber nur in besonders ausgewählten Fällen zur Radikaloperation, wir haben sie nur in sehr selt e nen Fällen nötig. Viel einfacher ist unsere konservative Behandlung, die den Vorteil der Ungefährlichkeit hat und kleine Narben erzielt, die kosmetisch kaum entstellen. 
Sind die Drüsen bereits vereitert, so geschieht die Behandlung nach den angegebenen Richtlinien.

Sind die tuberkulösen Drüsen nur verkäst und geschlossen, so bringt man sic am besten durch intramuskuläre Einspritzung von Ol. terebinthinae zur Heilung. Man spritzt zunächst $0,3 \mathrm{~T}$ crpentinöl cin und beobachtet, wie der Kranke hierauf reagiert. Tritt keine heftigere Reaktion auf, so kann dic Einspritzung jede Woche zweimal bis zur Heilung wiederholt werden. Bei konsequenter Behandlung sind bisher alle Kranken, bei denen die Be handlung als abgeschlossen gelten kann, zur Heilung gckommen. Ëine andere Behandlung als die genannte wurde bei Halsdrüsen. tuberkulose nicht durchgeführt.

Auch die Spinac ventosae sowohl der Hand als des FuBes heilen, solange sie geschlossen sind, allein unter Einspritzung von Terpentinöl. Bestehen bereits Fisteln, so werden sic nach den oben angegebenen Regeln behandelt. Die Tuberkulosen der Wirbelsäule werden am besten im Streckverband unter regelmäßiger Einspritzung von Terpentinöl in die Gesäßmuskulatur behandelt. 